\title{
Basal or stress-induced cortisol and asthma development: the TRAILS study
}

\author{
Nienke M. Vink*,\#, H. Marike Boezen*,\#, Dirkje S. Postma ${ }^{\#, \oplus}$ and \\ Judith G.M. Rosmalen ${ }^{+}$
}

\begin{abstract}
We examined the association between: 1) cortisol levels and asthma or asthma development; 2) cortisol levels upon stress and asthma. In addition, we performed a post hoc meta-analysis on results from the literature.
\end{abstract}

Cortisol, cortisol upon stress, asthma (doctor diagnosis of asthma and/or symptoms and/or treatment in the past 12 months) and asthma development (asthma at a specific survey while not having asthma at the previous survey(s)) were assessed in the TRAILS study $(n=2230$, mean age at survey 111 years, survey 214 years and survey 316 years). Logistic regression models were used to study associations between: 1) cortisol (cortisol awakening response, area under the curve (AUC) with respect to the ground (AUCg) or with respect to the increase (AUCi), and evening cortisol) and asthma or asthma development; 2) cortisol upon stress (AUCg or AUCi) and asthma. The meta-analyses included nine case-control articles on basal cortisol in asthma.

No significant association was found between: 1) cortisol and asthma (age 11 years) or asthma development (age 14 or 16 years); 2) cortisol upon stress and asthma (age 16 years). The metaanalysis found lower morning and evening cortisol levels in asthmatics compared to nonasthmatics; however, the summary estimates were not significant.

We found no evidence supporting a role for cortisol in asthma and asthma development.

KEYWORDS: Adolescents, asthma, cortisol, hypothalamic-pituitary-adrenal axis, population cohort

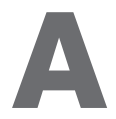
sthma is a chronic inflammatory airway disorder, with many pathways involved in its aetiology. Psychosocial stress can trigger asthma exacerbations [1] and it is suggested that psychosocial stress is also involved in asthma development [2-6]. Psychosocial stress activates the hypothalamic-pituitary-adrenal (HPA) axis leading to an increase in cortisol secretion on top of the circadian rhythm of cortisol secretion [7]. Cortisol influences the activity of many systems in the human body, including the immune system [8]. Since cortisol induces a shift in the T-helper cell 1 (Th1)/Th2 balance of peripheral blood mononuclear cells towards a predominantly Th2 response [7], an alteration in HPA axis function is suggested to be one of the potential mechanisms via which psychosocial stress leads to asthma development.

Several cross-sectional studies have investigated cortisol levels in asthmatics and non-asthmatics, with diverse results. Not only have lower [9-11] or normal cortisol levels [12-16] been reported in asthmatics compared to non-asthmatics, but also higher $[17,18]$ cortisol levels have been reported. Since all these studies investigated different aspects of the circadian pattern of cortisol secretion in relation to asthma, their results are incomparable.

Because psychosocial stress triggers asthma exacerbations [1], it could be argued that dysfunctions of the HPA axis become more evident under stressful conditions. One previous study investigated both basal cortisol levels and cortisol responses to a laboratory stress task (Trier Social Stress Test) in asthmatics and non-asthmatics [12]. Although this study found no differences in basal cortisol levels between asthmatics and non-asthmatics, cortisol levels in response to a laboratory stress task were lower in asthmatics compared to non-asthmatics, indicating hyporesponsiveness of the HPA axis which only became evident under exposure to stress. Because of the small sample size of this study (asthmatics $n=17$, non-asthmatics $n=18$ ) these findings need to be replicated in a larger sample size.

\section{AFFILIATIONS}

*Dept of Epidemiology, University of Groningen, University Medical Center Groningen, Groninger Research Institute for Asthma and COPD, Groningen,

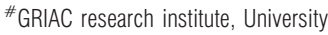
of Groningen, University Medical Center Groningen, Groningen, "Dept of Pulmonology, University of Groningen, University Medical Center Groningen, Groninger Research Institute for Asthma and COPD, Groningen, and

${ }^{+}$Interdisciplinary Center for Psychopathology and Emotion regulation, University of Groningen, University Medical Center Groningen, Groningen, The Netherlands.

\section{CORRESPONDENCE}

J.G.M. Rosmalen

Interdisciplinary Center for

Psychopathology and Emotion regulation, University of Groningen University Medical Center Groningen Hanzeplein 1

P0 Box 30001

9700 RB Groningen

The Netherlands

E-mail: j.g.m.rosmalen@umcg.nl

Received:

Feb 032012

Accepted after revision:

June 202012

First published online:

July 122012 
In conclusion, results from previous cross-sectional studies have suggested that asthmatics have an altered HPA axis function. However, it is unclear whether this alteration in HPA axis function precedes the development of asthma or is the result of asthma. To our knowledge, no previous longitudinal study has been performed to investigate alterations in HPA axis function as an aetiologic mechanism contributing to the development of asthma. We hypothesised that: 1) there is a cross-sectional association between low cortisol and asthma; 2) low cortisol levels precede the development of asthma; and 3) adolescents with asthma have a blunted cortisol response upon exposure to stress.

\section{MATERIALS AND METHODS Study participants}

The Tracking Adolescents' Individual Lives Survey (TRAILS) is a prospective cohort study among Dutch adolescents. A detailed description of the sampling procedure and methods has been published previously [19]. Briefly, the TRAILS target sample involved 10-12-year-olds (born between October 1, 1989 and September 30, 1990 for the first two municipalities or between October 1, 1990 and September 30, 1991 for the last three municipalities) living in five municipalities in the north of the Netherlands, including both urban and rural areas. A total of 135 primary schools were invited to participate, encompassing 3483 eligible children. Of the 135 schools 13 refused to participate, resulting in the exclusion of 338 children. Of the 3145 remaining eligible children, 210 were excluded because they were either unable to participate or incapable of participating due to severe mental retardation or due to a serious physical illness or handicap, or if no Dutch-speaking parent or parent surrogate was available (Turkish and Moroccan parents who were unable to speak Dutch were interviewed in their own language). After intensive recruitment efforts (including telephone calls, reminder letters and home visits), a total of 2230 children $(76.0 \%)$ were included in the study at baseline. So far, three assessment surveys have been completed $(n=2230$, mean \pm SD age $11 \pm 0.6$ years; $n=2149,14 \pm 0.5$ years; $n=1816$, $16 \pm 0.7$ years)

\section{Cortisol collection at age 11 years}

Cortisol was assessed from saliva taken at three times during one day: shortly after waking up (Cort07.00); 30 minutes after waking up (Cort07.30); and at $20.00 \mathrm{~h}$ (Cort20.00) at age 11 years. Saliva samples were received from 1768 adolescents (details on collection and assays have been published previously [20]). Non-responders did not differ from responders in terms of sex; non-responders were slightly older (mean age 11.2 versus 11.1 years) [20].

\section{Stress test and cortisol collection at age 16 years}

At age 16 years, 715 adolescents performed a stress test, based on (but not identical to) the Trier Social Stress Task [21]. The stress test consisted of two parts. In the first part, the adolescents were instructed to prepare a 6-min speech about themselves and their lives and deliver this speech in front of a video camera. The speech was followed by a 3-min interlude in which the adolescents were not allowed to speak. In the second part, adolescents were asked to perform a 6-min mental arithmetic task. The adolescents were instructed to repeatedly subtract the number 17 from a larger sum, starting with 13 287. The mental arithmetic task was followed by a 3-min period of silence, after which the adolescents were debriefed about the experiment. Adolescents with a high risk of mental health problems were over-represented in this population (details in the online supplementary material).

Cortisol was assessed from saliva collected prior to the stress test (Cort1), directly after (Cort2), and 20 minutes (Cort3) and 40 minutes (Cort4) after the stress test (details on collection and assays have been published previously [22]). Non-responders did not differ from responders in terms of sex; non-responders were slightly older (mean age 16.4 versus 16.1 year) [23].

\section{Asthma}

Data on asthma were collected via questionnaires at age 11, 14 and 16 years [24]. Asthma was defined as having a doctor diagnosis (Did a physician give your child a diagnosis of asthma?) (assessed at age 11 years) and/or symptoms and/or treatment for asthma in the past 12 months (assessed at all surveys). Asthma development was defined as having asthma at a specific survey, while not having asthma at all previous surveys.

\section{Statistical analysis}

Area under the curve with respect to the ground (AUCg) (11 years), AUCg (stress-induced), area under the curve with respect to the increase (AUCi) (11 years) and AUCi (stressinduced) were calculated (formulae are in the online supplementary material) $[20,25,26]$. To correct for skewed distributions, cortisol values above or below $3 \times \mathrm{SD}$ of the mean were regarded as outliers and excluded, after which all cortisol values were transformed to z-scores to normalise the data in order to be able to compare results on different cortisol measures. Including adolescents with cortisol values below or above $3 \times$ SD of the mean in the analysis did not change the results.

Logistic regression analyses were used to study associations between basal cortisol and asthma at age 11 years and between basal cortisol and asthma development at age 14 and 16 years. All analyses were adjusted for sex and quadratic effect of sampling month, since these are known to be associated with cortisol values [20] and/or asthma [24] in this cohort.

Furthermore, logistic regression analyses were used to study associations between cortisol response to stress test and asthma at age 16 years, adjusted for sex and sampling weights to correct for the oversampling of adolescents with a high risk of mental health problems, and in case of AUCi (stressinduced) also for baseline cortisol level (Cort1) (online supplementary material).

All analyses were repeated adjusting for depression (affective problems scale of the Youth Self-Report [27]), physical activity and smoking in case of basal cortisol, and additionally adjusting for age, body mass index (BMI) and oral contraceptive use in case of stress-induced cortisol. A previous study in this cohort showed that age and BMI were not related to basal cortisol [20].

To examine the impact of (inhaled and oral) steroid treatment on the above studied associations, sensitivity analyses were performed by excluding adolescents who used corticosteroid 
medication at age 11 years, in case of basal cortisol, or at age 16 years, in case of stress-induced cortisol.

Statistical analyses were performed using SPSS Version 18.0 (SPSS Inc., Chicago, IL, USA). p-values $<0.05$ (tested twosided) were considered to be significant.

\section{RESULTS}

\section{Study population}

Table 1 shows the characteristics of the study populations stratified according to asthma at age 11 years. $22(1 \%)$ adolescents used corticosteroid-containing medication. There was no significant difference in age, sex and cortisol levels between adolescents with and without asthma at age 11 years. However, adolescents with asthma used significantly more corticosteroid-containing medication compared to adolescents without asthma.

In 489 (30\%) adolescents, the AUCi (11 years) was negative (cortisol levels measured at $07.00 \mathrm{~h}$ were higher compared to cortisol levels measured at $07.30 \mathrm{~h}$ ). At age 11 years, no significant differences were found between adolescents with a positive or a negative AUCi with respect to the proportion of adolescents with asthma: 34 out of $474(7 \%)$ adolescents with a negative AUCi had asthma versus 73 out of $1122(7 \%)$ adolescents with a positive AUCi had asthma (Chi-squared $=0.24, \mathrm{p}=0.63)$.

\section{Cross-sectional associations between cortisol levels at age 11 years and asthma}

Logistic regression analyses, adjusted for sex and quadratic effect of sampling month, showed no significant cross-sectional associations between asthma at age 11 years and AUCg (11 years), AUCi (11 years) or Cort20.00 h (table 2). Adjusting for depression, physical exercise and smoking did not essentially affect the results. In addition, excluding adolescents using corticosteroid-containing medication did not essentially affect the results.

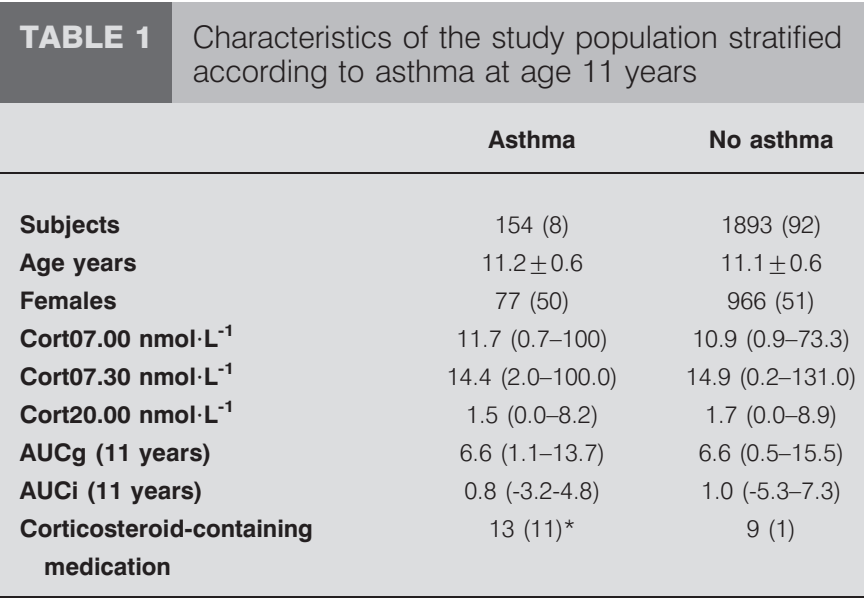

Data are presented as $\mathrm{n}(\%)$, mean \pm SD or median (range). Cort07.00: cortisol measured shortly after waking up; Cort07.30: cortisol measured 30 min after waking up; Cort20.00: cortisol measured at $20.00 \mathrm{~h}$; AUCg: area under the curve with respect to the ground at age 11 years; $\mathrm{AUCi}$ : area under the curve with respect to the increase at age 11 years. * $p<0.05$

\section{Longitudinal association between cortisol levels at age} 11 years and asthma development at age 14 or 16 years Logistic regression analyses, adjusted for sex and quadratic effect of sampling month, showed no significant association between AUCg (11 years), AUCi (11 years) or Cort20.00, and asthma development at age 14 years, or age 16 years (table 2). Adjusting for depression, physical exercise and smoking did not essentially affect the results. In addition, excluding adolescents using corticosteroid-containing medication did not essentially affect the results.

\section{Study population stress-induced cortisol}

Table 3 presents the characteristics of the study population participating in the stress experiments, stratified according to asthma at age 16 years. 34 (5\%) adolescents used corticosteroidcontaining medication. There was no significant difference in age, sex and cortisol levels between adolescents with and without asthma at age 16 years. Adolescents with asthma used significantly more corticosteroid-containing medication compared to adolescents without asthma.

\section{Cross-sectional association between cortisol levels during the stress test and asthma at age 16 years}

Logistic regression analysis, adjusted for sex and sampling weights to correct for the oversampling of adolescents with a high risk of mental health problems, showed no significant association between AUCg (stress-induced) and asthma at age 16 years (OR 0.94, 95\% CI 0.67-1.31). Comparable results were found for the association between AUCi (stress-induced) and asthma at age 16 years (OR 1.04, 95\% CI 0.74-1.47). Adjusting for age, depression, physical exercise, smoking, BMI and OC use did not essentially affect the results. In addition, excluding adolescents using corticosteroid-containing medication did not essentially affect the results.

\section{DISCUSSION}

The present study did not show an association between basal or stress-induced cortisol levels and asthma when analysing the data cross-sectionally. Furthermore, we found no association between basal cortisol levels and the development of asthma in the longitudinal analyses.

This is the first study that investigated associations between basal cortisol levels and asthma in a large study population ( $n=2230$ adolescents). We found no association between basal cortisol measured at age 11 years and asthma at age 11 years. Previous studies have shown lower [9-11], comparable [12-16] and higher $[17,18]$ cortisol levels in asthmatics than nonasthmatics. Therefore, we have performed a post hoc metaanalysis to obtain a stronger conclusion about the association between cortisol and asthma. To allow pooling across studies that used different types of HPA axis measurements, we calculated a standardised mean difference with $95 \%$ confidence intervals of basal cortisol levels in the morning and basal cortisol levels in the evening (see online supplementary tables E1 and E2) [28]. Both morning and evening cortisol levels were lower in asthmatics than non-asthmatics; however, the summary estimates were not significant (fig. 1 and 2). Funnel plots of the morning and evening cortisol levels indicated a possible publication bias (see online supplementary fig. E1 and E2), suggesting unidentified unpublished articles, which are mostly negative or neutral articles. This potential 
TABLE 2 Estimated odds ratios (95\% confidence intervals) of the association between basal cortisol measured at age 11 years and asthma or asthma development adjusted for sex and quadratic effect of sampling month

Asthma at age 11 years

Asthma development at age 14 years
Asthma development at age 16 years

$\begin{array}{llrr}\text { AUCg (11 years) } & 0.99(0.80-1.23) & 1.46(0.97-2.19) & 1.27(0.76-2.12) \\ \text { AUCi (11 years) } & 0.91(0.73-1.12) & 1.14(0.74-1.76) & 1.06(0.63-1.76) \\ \text { Cort20.00 nmol } \cdot \mathrm{L}^{-1} & 0.89(0.71-1.11) & 1.26(0.85-1.86) & 1.12(0.67-1.86)\end{array}$

Values are shown as z-scores. AUCg: area under the curve with respect to the ground at age 11 years; AUCi: area under the curve with respect to the increase at age 11 years; Cort20.00: cortisol measured at $20.00 \mathrm{~h}$ at age 11 years.

publication bias would be compatible with the fact that our study, despite having the largest sample size published, failed to find any significant association between cortisol and asthma or asthma development. It again underlines the importance of publishing studies that report the absence of a significant association, especially those with large sample sizes.

This study is the first one investigating the research question of whether alterations in HPA-axis function precede asthma development. Neither asthma development at age 14 years, nor asthma development at age 16 years was significantly associated with basal cortisol levels upon awakening or evening cortisol level measured at age 11 years. Therefore, we did not find evidence that an alteration in HPA axis function precedes asthma development between ages 14 and 16 years. However, this study cannot rule out that alterations in HPA axis function precede adult-onset asthma.

Contrary to a previous study that showed a blunted cortisol response to stress in asthmatics [12], our study did not find such an association. Differences in study population and sample sizes could explain these inconsistent findings. The previous study included children $(n=35$, age $7-13$ years), whereas our study included adolescents ( $n=715$, age $15-17$ years). Other explanations could involve differences in the time points at which cortisol was measured, and differences in the way the associations between cortisol levels to a stress task and asthma were studied. The previous study measured cortisol 35, 25, 15 and 1 min before, and 10,20,30 and 40 min after the stress test and compared individual time points between asthmatic and healthy children. Our study measured cortisol at the start, directly after, and 20 and $40 \mathrm{~min}$ after the end of the stress test and investigated whether AUC was associated with asthma. When investigating individual cortisol levels measured at 20 and $40 \mathrm{~min}$ after the stress test in our study, we found no differences in cortisol levels between asthmatics and nonasthmatics $(\mathrm{U}=12$ 731.0, $\mathrm{p}=0.98$ and $\mathrm{U}=11$ 453.0, $\mathrm{p}=0.39$, respectively). Therefore, our data suggest that cortisol responses upon exposure to stress are not associated with asthma.

The strength of this study is its longitudinal design, allowing us to study the effect of cortisol prior to the onset of asthma. In addition, our study was performed in a large sample of the general population, thereby increasing the probability that our findings with respect to basal cortisol levels are generalisable to the population at large. However, this advantage of the large sample size comes with some associated disadvantages, especially the level of detail that was reached in the phenotyping of asthma and in the characterisation of HPA axis activity in our study. The asthma population in our study is heterogeneous in terms of phenotype, severity and management. Therefore, we may have missed potential associations with cortisol in specific subgroups such as those with nocturnal asthma or allergic asthma. We do not have information about whether adolescents

\section{TABLE 3 Characteristics of the population participating in the stress test according to asthma at age 16 years}

\begin{tabular}{|c|c|c|}
\hline Subjects & $42(6)$ & 621 (94) \\
\hline Females & $23(55)$ & $315(51)$ \\
\hline Cort1 nmol. $\mathrm{L}^{-1}$ & $3.0(0.3-10.8)$ & $3.2(0.0-70.1)$ \\
\hline Cort2 $\mathrm{nmol} \cdot \mathrm{L}^{-1}$ & $4.3(0.5-9.6)$ & $3.8(0.0-68.0)$ \\
\hline AUCg (stress-induced) & $226.3(56.0-588.0)$ & $234.0(16.5-855.8)$ \\
\hline AUCi (stress-induced) & $36.5(-244.0-372.5)$ & $16.5(-359.5-448.3)$ \\
\hline Corticosteroid-containing medication & $22(52)^{*}$ & $11(2)$ \\
\hline
\end{tabular}




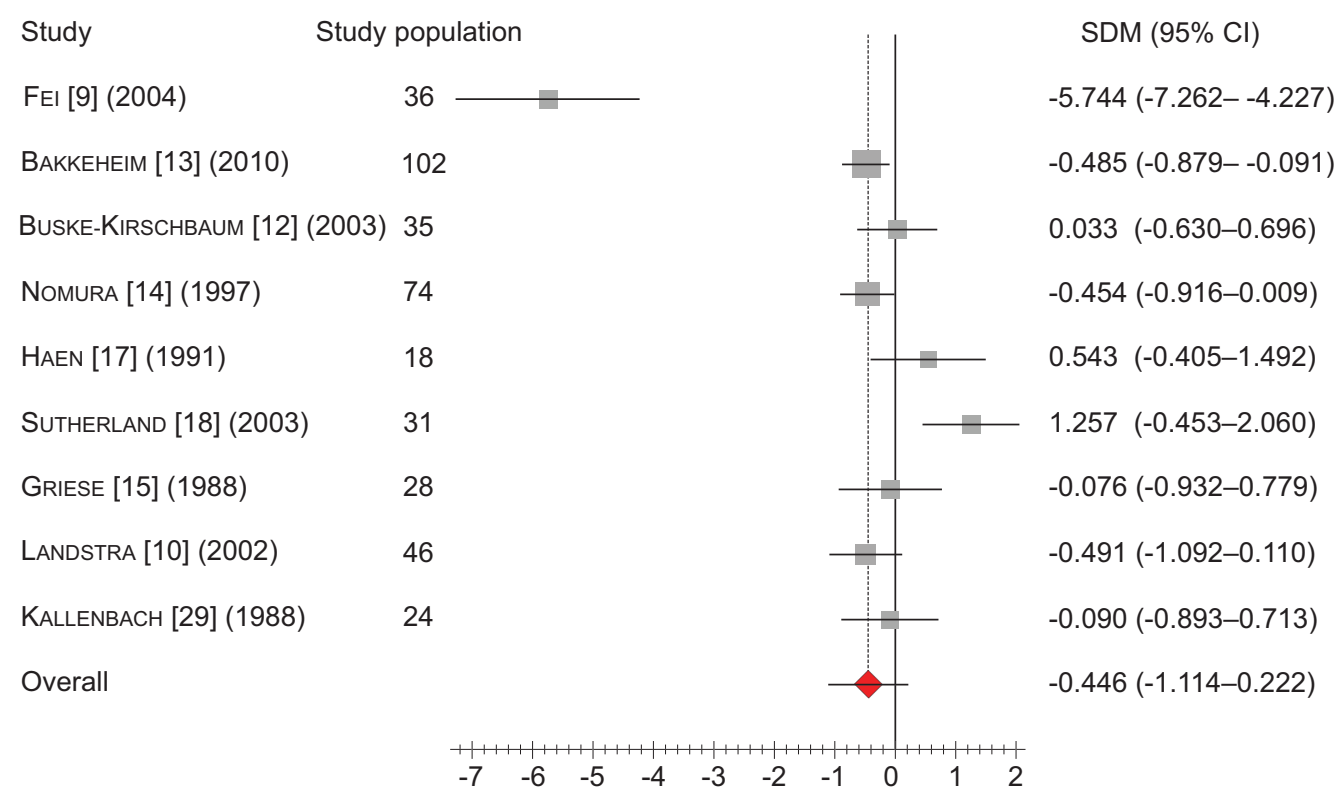

FIGURE 1. Forest plot of the standardised mean differences (SDM) in morning cortisol levels in asthmatics and non-asthmatics. Test for heterogeneity $Q$-test Chi-square $=70.04$, degrees of freedom $=8, p<0.0001 ; \mathrm{I}^{2}=0.89$.

with asthma have allergic or non-allergic asthma. However, we do have information about the presence of allergy, hay fever and eczema, which made it possible to give some indication whether asthmatic adolescents in our study have allergic (asthma with allergy, hay fever or eczema) or non-allergic asthma (asthma without allergy, hay fever and eczema). Sensitivity analysis revealed comparable associations with cortisol for allergic and non-allergic asthma (data not shown).
With respect to the characterisation of the HPA axis activity, cortisol was assessed at 1 day in our study. Previous studies showed that cortisol levels have notable intra-individual variability $[12,16]$, which is reduced when sampling on a workday, as a result of the usually strict schemes [30]. In our study, cortisol was mostly assessed on school days, which are highly scheduled as well, so we expect that the same reduction in intra-individual variability applies to our adolescents. In

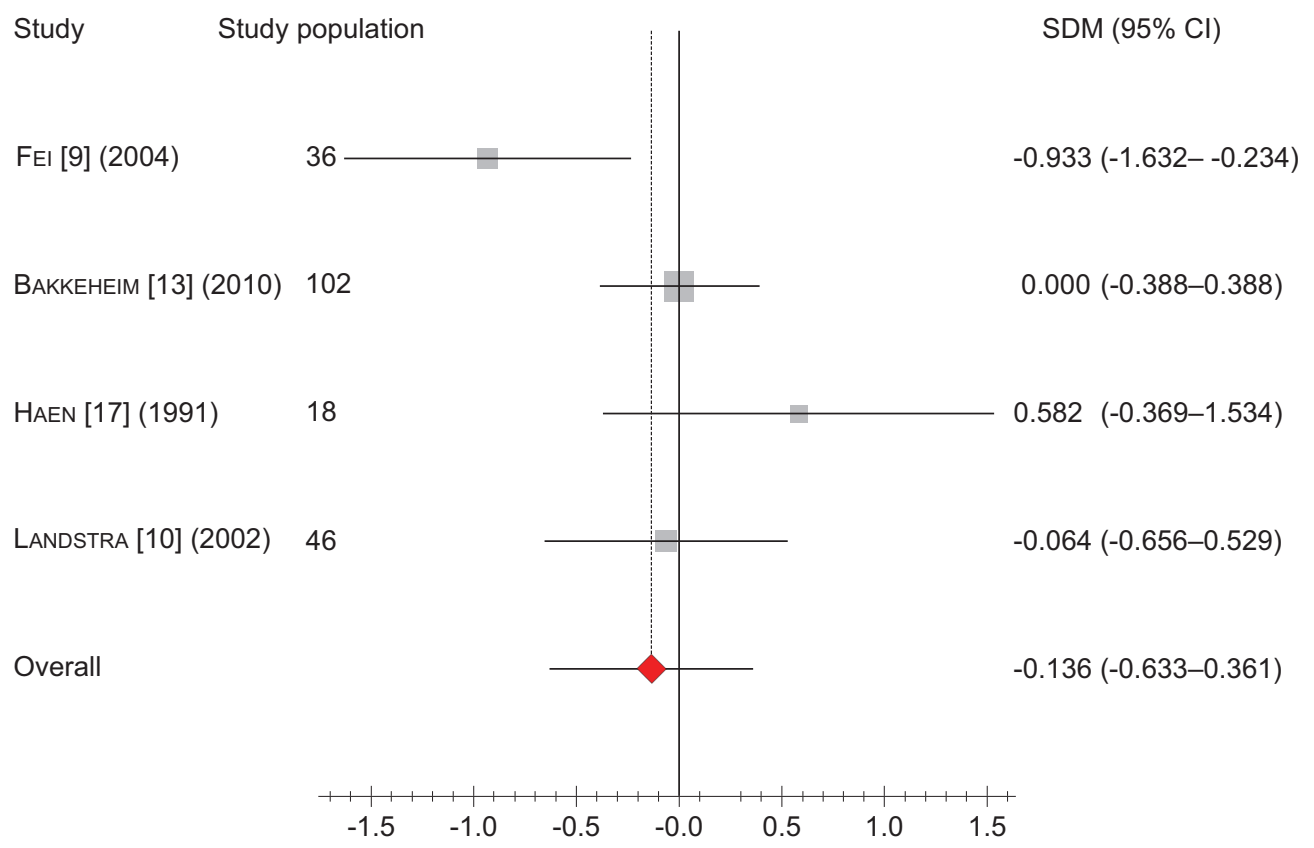

FIGURE 2. Forest plot of the standardised mean differences (SDM) in evening cortisol levels in asthmatics and non-asthmatics. Test for heterogeneity Q-test Chisquared $=7.70$, degrees of freedom $=3, p=0.05 ; 1^{2}=0.61$. 
addition, we expect that the large sample size will compensate for the possible reduction in reliability as a result of one day cortisol assessment, so random fluctuations in individual values will be set off.

In our study, cortisol was assessed at three time points, namely shortly after waking up (still lying in bed), 30 min after waking up and at $20.00 \mathrm{~h}$. As a consequence only the cortisol awakening response and cortisol levels at $20.00 \mathrm{~h}$ could be studied in relation to asthma and asthma development, and not the circadian rhythm of the cortisol secretion. Therefore, we cannot rule out that there is an association between specific elements of this circadian rhythm of cortisol secretion and asthma presence or development.

We were unable to find evidence suggesting a role for cortisol in asthma, despite its well-established anti-inflammatory properties and therapeutic effects when given as inhaled or oral corticosteroids. The question arises whether our study indicates a real lack of association, or whether it merely reflects the problems associated with large cohort studies. Further prospective studies are needed to unravel the role of cortisol in asthma or asthma development. It is pivotal for such studies that both cortisol and asthma are measured in detail. For cortisol, this implies measuring multiple time points and multiple days, in order to obtain a reliable and robust estimate of HPA axis activity. For asthma, detailed phenotyping would enable the study of differences in the association with cortisol in relation to asthma severity and medication use, and also in relation to subtypes, including glucocorticoid resistant asthma or non-allergic asthma. Complaints of nocturnal worsening are especially interesting because of the circadian rhythm of cortisol secretion and the anti-inflammatory effect of cortisol.

In summary, our study did not find evidence supporting a role for cortisol in asthma and asthma development. In addition, our study found no association between stress-induced cortisol levels and asthma. Further longitudinal studies from birth onward have to assess whether there exists a window of time in which alterations in HPA axis function may contribute to asthma development. However, in adolescence, it seems unlikely that this plays a dominant role.

\section{SUPPORT STATEMENT}

This research was funded by a grant of the Netherlands Asthma Foundation (grant no 3.4.07.034). TRAILS has been financially supported by various grants from the Netherlands Organization for Scientific Research NWO (Medical Research Council program grant GB-MW 940-38-011; ZonMW Brainpower grant 100-001-004; ZonMw Risk behaviour and Dependence grants 60-60600-97-118; ZonMw Culture and Health grant 261-98-710; Social Sciences Council mediumsized investment grants GB-MaGW 480-01-006 and GB-MaGW 480-07001; Social Sciences Council project grants GB-MaGW 452-04-314 and GB-MaGW 452-06-004; NWO large-sized investment grant 175.010.2003.005; NWO Longitudinal Survey and Panel Funding 48108-013), the Dutch Ministry of Justice (WODC), the European Science Foundation (EuroSTRESS project FP-006), Biobanking and Biomolecular Resources Research Infrastructure BBMRI-NL (CP 32), and the participating universities.

\section{STATEMENT OF INTEREST}

Conflict of interest information can be found alongside the online version of this article at www.erj.ersjournals.com

\section{ACKNOWLEDGEMENTS}

This research is part of the TRacking Adolescents' Individual Lives Survey (TRAILS). Participating centres in TRAILS include: various departments of the University Medical Center and University of Groningen; the Erasmus University Medical Center Rotterdam; the University of Utrecht; the Radboud Medical Center Nijmegen; and the Parnassia Bavo group, all in the Netherlands. We are grateful to all adolescents, their parents and teachers who participated in this research and to everyone who worked on this project and made it possible.

\section{REFERENCES}

1 Chida Y, Hamer M, Steptoe A. A bidirectional relationship between psychosocial factors and atopic disorders: a systematic review and meta-analysis. Psychosom Med 2008; 70: 102-116.

2 Cookson H, Granell R, Joinson C, et al. Mothers' anxiety during pregnancy is associated with asthma in their children. J Allergy Clin Immunol 2009; 123: 847-853.

3 Kilpelainen M, Koskenvuo M, Helenius H, et al. Stressful life events promote the manifestation of asthma and atopic diseases. Clin Exp Allergy 2002; 32: 256-263.

4 Kozyrskyj AL, Mai XM, McGrath P, et al. Continued exposure to maternal distress in early life is associated with an increased risk of childhood asthma. Am J Respir Crit Care Med 2008; 177: 142-147.

5 Subramanian SV, Ackerson LK, Subramanyam MA, et al. Domestic violence is associated with adult and childhood asthma prevalence in India. Int J Epidemiol 2007; 36: 569-579.

6 Suglia SF, Enlow MB, Kullowatz A, et al. Maternal intimate partner violence and increased asthma incidence in children: buffering effects of supportive caregiving. Arch Pediatr Adolesc Med 2009; 163: 244-250.

7 Elenkov IJ, Chrousos GP. Stress hormones, Th1/Th2 patterns, pro/anti-inflammatory cytokines and susceptibility to disease. Trends Endocrinol Metab 1999; 10: 359-368.

8 Elenkov IJ, Chrousos GP. Stress system - organization, physiology and immunoregulation. Neuroimmunomodulation 2006; 13: 257-267.

9 Fei GH, Liu RY, Zhang ZH, et al. Alterations in circadian rhythms of melatonin and cortisol in patients with bronchial asthma. Acta Pharmacol Sin 2004; 25: 651-656.

10 Landstra AM, Postma DS, Boezen HM, et al. Role of serum cortisol levels in children with asthma. Am J Respir Crit Care Med 2002; 165: 708-712.

11 Kauffmann F, Guiochon-Mantel A, Neukirch F. Is low endogenous cortisol a risk factor for asthma? Am J Respir Crit Care Med 1999; 160: 1428 .

12 Buske-Kirschbaum A, von Auer K, Krieger S, et al. Blunted cortisol responses to psychosocial stress in asthmatic children: a general feature of atopic disease? Psychosom Med 2003; 65: 806-810.

13 Bakkeheim E, Mowinckel P, Carlsen KH, et al. Reduced basal salivary cortisol in children with asthma and allergic rhinitis. Acta Paediatr 2010; 99: 1705-1711.

14 Nomura S, Fujitaka M, Sakura N, et al. Adrenocortical function in asthmatic children: low levels of adrenocortical hormones in children with persistent attacks. Eur J Pediatr 1997; 156: 323-328.

15 Griese M, Kusenbach G, Lusebring K, et al. Glucocorticoid receptors in mononuclear blood cells and their correlation to endogenous and exogenous corticoids in healthy and asthmatic children. Eur J Pediatr 1988; 147: 490-495.

16 Wolf JM, Nicholls E, Chen E. Chronic stress, salivary cortisol, and alpha-amylase in children with asthma and healthy children. Biol Psychol 2008; 78: 20-28.

17 Haen E, Hauck R, Emslander HP, et al. Nocturnal asthma. Beta 2adrenoceptors on peripheral mononuclear leukocytes, cAMP- and cortisol-plasma concentrations. Chest 1991; 100: 1239-1245. 
18 Sutherland ER, Ellison MC, Kraft M, et al. Altered pituitaryadrenal interaction in nocturnal asthma. J Allergy Clin Immunol 2003; 112: 52-57.

19 Huisman M, Oldehinkel AJ, de Winter A, et al. Cohort profile: the Dutch 'TRacking Adolescents' Individual Lives' Survey'; TRAILS. Int J Epidemiol 2008; 37: 1227-1235.

20 Rosmalen JGM, Oldehinkel AJ, Ormel J, et al. Determinants of salivary cortisol levels in 10-12 year old children; a populationbased study of individual differences. Psychoneuroendocrinology 2005; 30: 483-495.

21 Kirschbaum C, Pirke KM, Hellhammer DH. The "Trier Social Stress Test" - a tool for investigating psychobiological stress responses in a laboratory setting. Neuropsychobiology 1993; 28: 76-81.

22 Bouma EM, Riese H, Ormel J, et al. Adolescents' cortisol responses to awakening and social stress; effects of gender, menstrual phase and oral contraceptives. The TRAILS study. Psychoneuroendocrinology 2009; 34: 884-893.

23 Bosch NM, Riese H, Reijneveld SA, et al. Timing matters: Long term effects of adversities from prenatal period up to adolescence on adolescents' cortisol stress response. The TRAILS study. Psychoneuroendocrinology 2012; 37: 1439-1447.

24 Vink NM, Postma DS, Schouten JP, et al. Gender differences in asthma development and remission during transition through puberty: the TRacking Adolescents' Individual Lives Survey (TRAILS) study. J Allergy Clin Immunol 2010; 126: 498-504.

25 Pruessner JC, Kirschbaum C, Meinlschmid G, et al. Two formulas for computation of the area under the curve represent measures of total hormone concentration versus time-dependent change. Psychoneuroendocrinology 2003; 28: 916-931.

26 Janssens KA, Oldehinkel AJ, Verhulst FC, et al. Symptom-specific associations between low cortisol responses and functional somatic symptoms: the TRAILS study. Psychoneuroendocrinology 2012; 37: 332-340.

27 Achenbach TM, Dumenci L, Rescorla LA. DSM-oriented and empirically based approaches to constructing scales from the same item pools. J Clin Child Adolesc Psychol 2003; 32: 328-340.

28 Tak LM, Meijer A, Manoharan A, et al. More than the sum of its parts: meta-analysis and its potential to discover sources of heterogeneity in psychosomatic medicine. Psychosom Med 2010; 72: 253-265.

29 Kallenbach JM, Panz VR, Joffe BI, et al. Nocturnal events related to "morning dipping" in bronchial asthma. Chest 1988; 93: 751-757.

30 Hellhammer J, Fries E, Schweisthal OW, et al. Several daily measurements are necessary to reliably assess the cortisol rise after awakening: state- and trait components. Psychoneuroendocrinology 2007; 32: 80-86. 